\title{
Properties of the inner penumbra boundary and temporal evolution of a decaying sunspot (Corrigendum)
}

\author{
M. Benko ${ }^{1}$, S. J. González Manrique ${ }^{1,2,3}$, H. Balthasar ${ }^{4}$, P. Gömöry ${ }^{1}$, C. Kuckein ${ }^{4}$, and J. Jurčák ${ }^{5}$ \\ 1 Astronomical Institute, Slovak Academy of Sciences, 05960 Tatranská Lomnica, Slovak Republic \\ e-mail: mbenko@ta3.sk \\ 2 Instituto de Astrofísica de Canarias, Vía Láctea s/n, 38205 La Laguna, Tenerife, Spain \\ 3 Departamento de Astrofísica, Universidad de La Laguna, 38205 La Laguna, Tenerife, Spain \\ ${ }^{4}$ Leibniz-Istitut für Astrophysik Potsdam (AIP), An der Sternwarte 16, 14482 Potsdam, Germany \\ 5 Astronomical Institute of the Academy of Sciences, Fričova 298, 25165 Ondřejov, Czech Republic
}

A\&A 620, A191 (2018), https://doi.org/10.1051/0004-6361/201834296

Key words. Sun: photosphere - Sun: activity - methods: observational - methods: data analysis techniques: high angular resolution - errata, addenda

In our paper, we made a mistake in calculating the magnetic flux values. The penumbra starts with a value of $1.32 \times 10^{21} \mathrm{Mx}$ on August 29, 2011, and decreases to $0.58 \times 10^{21} \mathrm{Mx}$ on September 3,2011 . The umbra has an initial value of $0.90 \times 10^{21} \mathrm{Mx}$ and ends with a value of $0.55 \times 10^{21} \mathrm{Mx}$. The corrected values for the penumbra now more likely have a quadratic trend, although we do not exclude a linear one. The new curves are displayed in Fig. 1, which replaces Fig. 4 in the original paper.

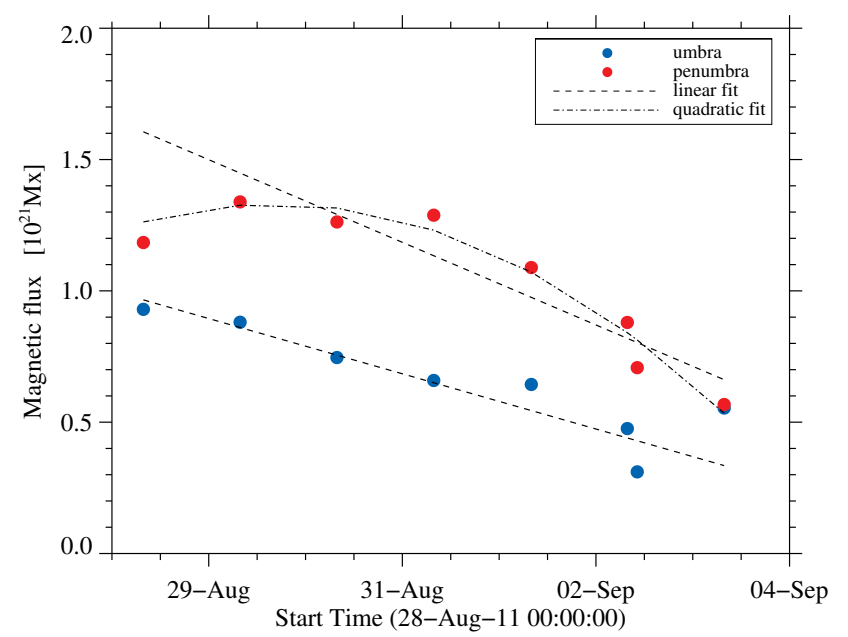

Fig. 1. Temporal evolution of the magnetic flux in umbra (blue dots) and penumbra (red dots). The data from August 28 are excluded from the fits because on that day the spot was still in the growing phase.

Acknowledgements. We are indebted to Dr. Mariarita Murabito for bringing the problem to our attention. 